\title{
Trying an IP Over NDN Packet Gateway
}

\author{
Marielena Márquez-Barreiro, Miguel Rodríguez-Pérez and Sergio Herrería-Alonso \\ atlanTTic Research Center \\ Universidade de Vigo \\ 36310 Vigo, Spain \\ Emails:mmarquez@alumnos.uvigo.es,miguel@det.uvigo.esandsha@det.uvigo.es
}

\begin{abstract}
Even though the TCP/IP architecture has served the Internet quite satisfactorily during its more than forty years of lifespan, there are doubts about whether this host-centric paradigm is well suited for the communication patterns of modern applications. These applications mainly need to get information pieces without being bothered about where they are located in the network. Additionally, the lack of both inbuilt security mechanisms and proper global multicast support may also be a symptom of fundamental problems with the classic architecture. Proponents of the novel Information Centric Networking (ICN) paradigm assert that the replacement of the TCP/IP architecture with one based on the information itself could be the ideal solution to all these problems. However, if such a replacement ever takes place, it will need the help of some transition mechanisms, for instance, for the transmission of legacy IP traffic on top of the new ICN based networks. In this paper we design and optimize such an open source IP over ICN transition application using the Named Data Networking (NDN) proposal as our target ICN network. We performed several tests that confirm that our prototype is able to transparently transport IP traffic across a pure NDN network with negligible packets losses, low jitter, and good performance.
\end{abstract}

\section{Introduction}

Nowadays, digital communications play a key role in almost every area of our society sustained by the common global telecommunication network known as the Internet. This network is based on the TCP/IP architecture, a layered protocol stack that allows applications to set up point-topoint channels to, in essence, transmit arbitrary information. Though this paradigm has served quite satisfactorily until now, it does not match well with the new usages and applications of the Internet, such as digital media, social networking, and smartphone applications. In fact, the IP requirement of discovering and specifying the source and the destination of each communication has become a nuisance for them. Therefore, there is a need to leave the original TCP/IP model (point-to-point communications, endpoints and addresses) behind and focus on what is relevant to current users and applications: the content itself.
In the last few years a radically different architecture for the network layer of the Internet has been gaining increased attention from the research community. Proposals under the Information Centric Networking (ICN) umbrella [1] enable consumer-driven communications where users can directly request information pieces, oblivious to their location. The Named Data Networking (NDN) project [2] is one of the most advanced proposals based on this architecture. It builds on principles adjusted to the new emerging communication patterns, thus making the network evolve from the current host-centric TCP/IP architecture to a more suited datacentric one. Nevertheless, the fact that all current devices and applications are designed according to the TCP/IP architecture assures that a potential transition towards NDN will be really challenging. Certainly, the Internet will have to face a transition period in which both TCP/IP and NDN models will coexist and, therefore, some tools will be required to ease the migration process. For instance, it is expected that the deployment of network gateways that enable the transport of IP traffic through an NDN network [3], [4] will receive a lot of attention in the next few years.

In this paper we present a new gateway design for efficient IP transmission across NDN realms. The development and proof of this kind of gateways requires performing the following set of tasks:

- The design of a forwarding protocol that enables the transport of IP packets using NDN as the network protocol.

- The implementation of an application that captures, processes and routes the IP traffic over NDN networks.

- The development of a testing environment to validate the gateway operations. For example, in this paper we will use a stringent streaming application to prove the successful transmission of IP data through the gateway.

The successful implementation of such an IP over NDN gateway would promote and streamline a future replacement of the current Internet network layer, thus transforming the present TCP/IP communication network into a true distribution network.

The rest of this paper is organized as follows. Section 2 provides a description of the main NDN mechanisms and describes some previous attempts to transmit IP packets through NDN networks. Section 3 describes the proposed 
IP over NDN gateway. Experimental results are detailed in Section 4. Finally, Section 5 summarizes our conclusions.

\section{Related Work}

In this Section we will first give a brief description of the NDN architecture. Then, we will discuss some existing literature about the transport of legacy (IP, TCP) traffic over NDN networks.

\subsection{Named Data Networking-NDN}

The Named Data Networking (NDN) project was founded in 2010 by the National Science Foundation (NSF) with the ambitious goal of becoming the new universal network layer of the Internet. The main service of the NDN network layer is fetching data. For this, every piece of data is given a unique name. This service is quite different from that of the Internet's original design, which was delivering packets to a given destination address. This fundamental change attempts to adjust the network layer to the needs of modern applications. While the only named entities in the IP packets are the communication endpoints, NDN removes this restriction so that any distributable object in an NDN network can be named.

In order to fulfill its service, the NDN network layer employs a communication protocol with two different type of packets: Interest and Data. Interest packets are sent by consumer applications to request a piece of named content from the network. The task of the network layer is to forward these Interest packets to any node capable of returning the requested content. Finally, the content is returned to the consumers inside a Data packet forwarded back by the NDN routers. Instead of the usual push-model of IP networks, where any node can enter new traffic in the network, the NDN exhibits a pull-model, as the consumers are the ones who trigger the communication process, since they are in charge of creating and sending an Interest packet to the network in the first place.

Despite the fact that NDN is still a developing project, it has already produced some interesting advances during the past few years. For instance, a formal protocol specification is available for all users, including a standardization of the format of NDN packets [5], a detailed description of the NDN link layer [6] and the documentation of multiple libraries to encourage the development of new NDN applications. In addition, the NDN Platform is also available for this purpose, which includes several tools such as the NDN Forwarding Daemon (NFD) [7], the $n d n$-cxx library [8] and the $n d n$-tools [9]. In fact, there are various applications that have already been deployed and tested. Among them, there are a few kinds that must be highlighted: video streaming [10], [11], real-time multiuser interaction [12], vehicular networking [13], etc. All of them have underlined and validated the major advantages of NDN over TCP/IP: a) its design oriented to content distribution; b) namespace design based on the particular needs of each application; c) robust and data-centric security; and d) support for mobility and broadcast.

\subsection{IP Over NDN}

The research literature about NDN already contains several approaches for the coexistence and migration of IP networks to NDN ones. According to [14], there are several alternative approaches for such a migration: $a$ ) a dual stack approach, akin to the one used for the IPv4 to IPv6 transition; b) a so-called hybrid approach, that performs address translation; and finally, $c$ ) a translation approach that devises gateways able to communicate between both stacks. These gateways may function at different levels of the communication stack. We are interested, in particular, in those translating gateways that perform network level translation so that any IP traffic can cross an NDN domain.

A few works have already explored this issue. [15] proposes and investigates different approaches to build a gateway. One of their alternatives is an straightforward networklayer gateway similar to what we will describe later as our first naive approximation to the problem in Section 3.1. However, such an approach suffers from poor performance and high transmission overhead. Other authors have tried more specialized gateways. For instance, in [16] the authors describe a proxying gateway for carrying TCP traffic on top of an ICN network. Another alternative based on translation is presented in [3]. This particular gateway, called dualchannel translation gateway, provides a privacy-preserving translation method that uses a name resolution table in order to securely bind an IP packet with a prefix name. The solution is appropriate for deploying content-centric applications in the IP realm, but, differently from ours, it provides no mechanisms for carrying general IP traffic across NDN only cores. Lastly, in [4] we find another work that opts for translation as the approach for IP to NDN migration. In this case, the translation method utilizes the data payload for bridging the semantic protocol gap in some possible producer and consumer scenarios. As before, it is assumed that the IP application is aware of the existence of the translation being carried out.

Taking all this into consideration, we consider that there is still an important need to fully fill the existing gap in the migration process from IP networks to NDN ones. And here is where our proposal of an IP over NDN gateway comes into play.

\section{Our IP over NDN Gateway}

Lets consider an example scenario as the one shown in Fig. 1 that will serve as a reference for the description of our gateway. ${ }^{1}$ The core of the network is composed of several routers that only employ the NDN protocol. There are also some hosts on different IP subnets that want to exchange IP traffic. Each IP subnet is connected to a gateway router

1. We have published the gateway code as free software, available for download at https://icarus.det.uvigo.es/ndnOverIP/. 


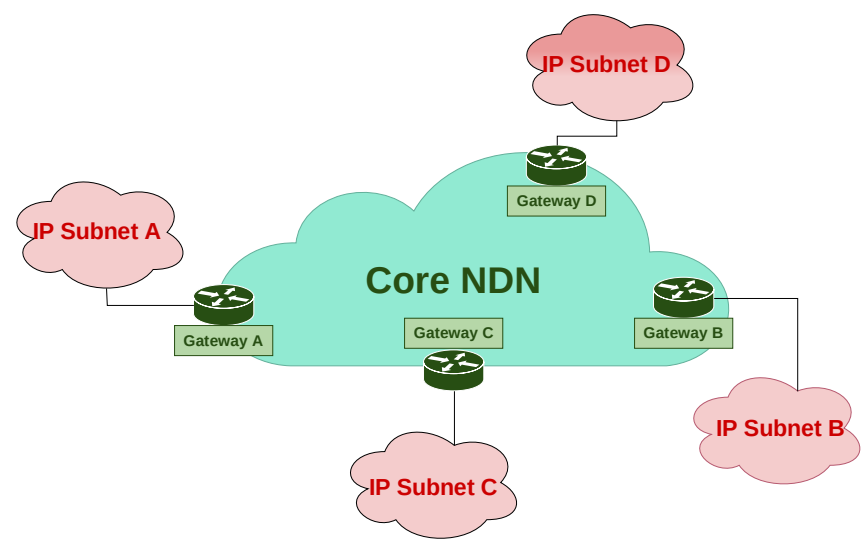

Figure 1. A core NDN network serving as the link layer for four IP subnets. Each router located at the edge of the NDN core must run an IP over NDN gateway.

that implements both the NDN and the IP architectures. In this way, IP packets can travel from one subnet to another one if the ingress gateway can forward the traffic across the NDN domain to the egress gateway connected to the destination subnet. For this scheme to work, each gateway must be the producer of a particular NDN prefix related to the address of the IP subnet to which it belongs. That is, it will produce named Data packets for IP datagrams captured by its IP interface when the destination address resides in an IP subnet connected to a different gateway. As we are only concerned with the design of the forwarding protocol, we will assume, for simplicity, that all the gateways know the routing table which lists the pairs of NDN gateways identifiers and the addresses of their directly connected IP subnets. This routing table dictates towards which gateway a specific IP packet must be forwarded according to its destination address. Thus, all the gateways will know which IP subnets are reachable through each gateway in the network.

To be able to forward IP traffic across the NDN core, the gateways must first capture the IP packets coming from the IP subnets they are connected to, so our tool also includes a traffic capture module implemented with the libpcap library [17]. This module sets up each network interface with a capture filter for IP traffic and it creates a process that waits for new IP packets to arrive. Each captured IP packet is processed to obtain its destination address and its length, which will be important when managing its forwarding through the NDN core. Then, if the forwarding table contains a next-hop gateway on the NDN core corresponding to the destination address, the packet is forwarded according to the protocol to be detailed below.

Once the IP packet arrives as the content of a Data packet at the corresponding egress gateway, our tool injects it as a raw IP packet in the destination subnet. The packet injection module of our tool uses RAW sockets, as they allow the direct transmission of IP packets without using any transport protocol and without specifying the link layer headers. In particular, the RAW sockets configured belong to the AF_INET family, which delegates the construction of

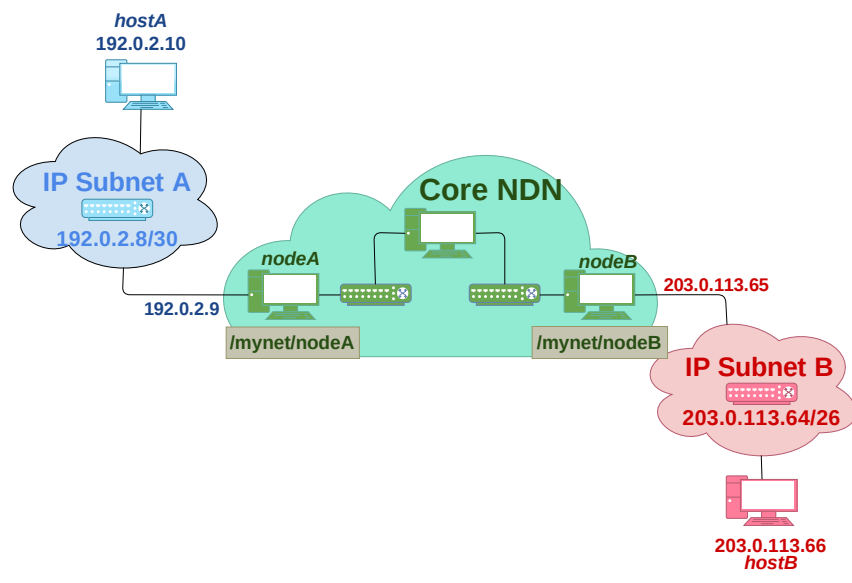

Figure 2. IP over NDN example scenario.

the link layer headers to the operating system itself. Furthermore, the protocol field specified during the creation of the sockets is IPPROTO_RAW, which means that, whenever the sendto function is called, the IP header itself is contained in its buffer parameter. Therefore, our module writes each IP header manually and the destination address included in it is the one used to forward the packet.

\subsection{Basic Forwarding Protocol}

As it was previously mentioned, the main task of the IP over NDN gateway is to solve the mismatch between the push model of the IP communication and the pull model of the NDN architecture. That is, a Data packet cannot be transmitted without a pending Interest packet in the network that has previously requested it. Thus, the design of this protocol must rely on one of the main NDN principles: communication is always triggered by a consumer. ${ }^{2}$ This mechanism is triggered whenever the gateway captures an IP packet and there is a matching entry for its destination address in the routing table. As a result, a gateway will not be able to send data (IP packets in this case) across the NDN core if it has not received a previous request from another gateway.

Having this constraint in mind, we propose a basic IP over NDN protocol involving three different stages which must be executed for each captured IP packet. In order to explain this protocol more clearly, we consider the particular scenario shown in Fig. 2 in which a host located in the IP subnet $B$ (referred to as hostB, with address 203.0.113.66) sends an IP packet to a host located in the IP subnet $A$ (referred to as hostA, with address 192.0.2.10). This particular case can be generalized to any scenario in which a host located in one of the IP subnets sends data to another host located in a different IP subnet. In the proposed scenario, the process shown in Fig. 3 takes place as follows:

2. The question about whether it is possible to send data inside Interest packets is a recurrent one in ICN forums. It is generally frowned upon as it would blur the distinction between both packet types and prevent the correct usage of the in-network caches, among other things. 


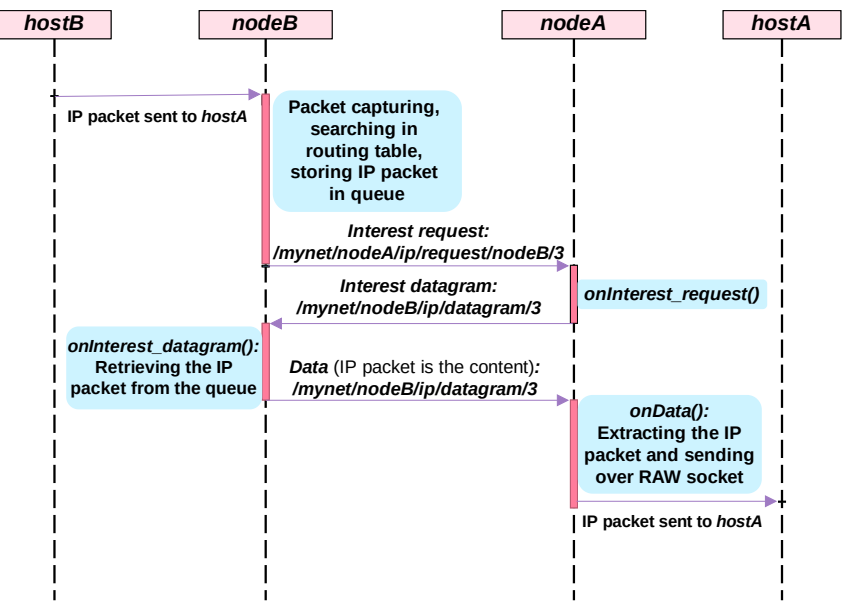

Figure 3. Time diagram of the basic gateway protocol.

1) The nodeB gateway captures the IP packet sent by host $B$ and searches for an entry in the routing table matching the corresponding IP destination address (192.0.2.10). Thus, nodeB finds out that hostA is reachable through the nodeA gateway. Then, node $B$ assigns a unique sequence number (seqno) to the IP packet and stores it in its pending packet queue. Note that a sequence number is required to identify each IP packet within a gateway since this could have several IP packets pending to be forwarded.

The next step is to notify nodeA of the existence of a new IP packet destined to it. For this, it sends an Interest packet asking for a specifically crafted name made up of the following strings: $a$ ) the identifier of the destination gateway obtained from the routing table; b) the string /ip/request/, where ip identifies the gateway process and request specifies the method; c) the identifier of the source gateway; and $d$ ) the sequence number assigned to the IP packet. We call this packet an Interest Request. In our example, if seqno $=3$, the name in the Interest Request would thus render /mynet/nodeA/ip/request/nodeB/3.

2) Thanks to the name in the Interest Request, this packet sent by nodeB is forwarded until it reaches nodeA, since there is no Data stored in the intermediary nodes that satisfies it. Then, nodeA executes onInterest_request(), a callback function registered during its initialization for the NDN prefix / mynet / <gateway identifier>/ip/request/. Within this callback, nodeA processes the Interest Request name and extracts the identifier of the source gateway and the sequence number assigned to the IP packet. With this information, nodeA sends a response Interest packet, which we will call Interest Datagram, with a name made up as the concatenation of the following substrings: a) the identifier of the gateway that sent the Interest Request and that will receive this Interest Datagram; b) /ip/datagram/, where, again, ip identifies the gateway process and datagram specifies the method; and c) the sequence number that was extracted from the Interest Request. Thus, in our example, the Interest Datagram is named as /mynet/nodeB/ip/datagram/3.

3) The Interest Datagram is forwarded until it finally reaches nodeB, since there is no Data packet stored in the network core that matches its name. Then, nodeB executes onInterest_datagram(), another callback method also registered during the gateway initialization, for the NDN prefix /mynet/<gateway identifier>/ip/datagram. This function processes the name of the Interest Datagram and extracts the sequence number that it carries. As a result, nodeB is able to identify the IP packet that this Interest Datagram authorizes to send and retrieves it from the pending packet queue. This IP packet becomes the content of the Data packet that nodeB sends to the network as response to the received Interest Datagram.

4) Finally, when nodeA receives the Data packet sent by nodeB, it executes the callback onData() to extract the IP packet that it contains. As a result, nodeA processes the IP header of this packet in order to know its destination address and length, and transmits it to its final destination on the subnet (hostA in our example).

\subsection{Improved Forwarding Protocol}

Although the basic protocol just described in the previous section works correctly, its performance is not very satisfactory since, as we will show in Section 4, it obtains too low transmission rates and significant packet losses. Note that, with this protocol, each Data packet enables the transport of only one IP packet. That is, the exchange of NDN packets among gateways is triggered once by each IP packet that is captured. Therefore, the transport of each IP packet involves the sending of three NDN packets through the core, thus greatly limiting the IP transmission rate.

To improve the system performance, the gateways must implement a new version of the protocol that reduces the number of NDN packets sent across the network core. For this, we can take advantage of the actual operation of current applications: IP packets typically belong to a transport layer connection and thus arrive in bursts or batches. Therefore, the design of the improved version of the protocol should take this time dependence into account.

Following this approach, the main enhancement in the new version is that now each Data packet that goes through the NDN core may contain more than a single IP datagram as long as all the datagrams being transported in the same Data packet satisfy that they share the same destination gateway. Note that, as in the basic protocol, the improved version also requires the exchange of three NDN packets, but the possibility of forwarding multiple IP packets into one Data packet will permit downsizing the frequency at which the exchange of the NDN packets occurs. As a result, the gateways must undergo the following changes:

- While in the basic version each gateway manages only one pending packet queue, in the new version each 
gateway must maintain one separate queue for each other gateway in the system. Moreover, each of these queues has its own independent sequence number space in order to identify its corresponding elements unequivocally.

- Since the content of each Data packet is no longer an individual IP packet but a collection of them, the elements of each queue are now called macro-packets. This term refers to the concatenation of multiple IP packets for the same destination gateway. Thus, in the new version, the content of each Data packet will be one macro-packet, consisting of a variable number of IP packets conditioned by their size. This restriction is set by the NFD itself (the canonical implementation of the NDN network layer), since it limits the maximum amount of data that can be transported in a Data packet.

These modifications do not require any changes to the capture and processing module of the incoming IP packets. Also, the format of the exchanged NDN packets remains unaffected except for the name used in the Interest Datagrams. Note that, in the new version, this name must also include the identifier of the source gateway in order to identify the queue where the named macro-packet is stored. The name carried by the Interest Datagram in our example would be thus /mynet/nodeB/ip/dagragram/nodeA/3, for the macro-packet with sequence number 3 to be sent from the ingress gateway node $B$ to the egress gateway nodeA.

However, the storage of the captured IP packets becomes more involved, since each gateway has now to manage multiple queues of macro-packets, instead of only one queue of individual IP packets. The storing process for each captured IP packet is shown in Fig. 4:

1) Firstly, the gateway finds out the queue in which the IP packet should be stored, according to the routing table.

2) Then, the gateway must determine if a new macropacket has to be created in order to store the IP packet just captured. This will only be required in three different cases: a) if the queue is empty; b) if the last macro-packet in this queue is already being processed in order to send it; or c) if appending this new IP packet to the end of the last macro-packet in the queue exceeds the size limit of a Data packet. Otherwise, it is not required to create a new macro-packet and the IP packet can be concatenated at the end of the last macro-packet in the queue.

3) Finally, the gateway has to decide if it is necessary to send an Interest Request to the network. The answer to this question will be yes only if a new macropacket was created in order to store the corresponding IP packet. If this was not the case, we know that an Interest Datagram will eventually arrive authorizing the sending of the macro-packet in which this IP packet was stored, since the corresponding Interest Request was already sent. Thus, there is no need to request it again by sending another Interest Request. Thanks to this logic, we will avoid exchanging three new NDN packets through the network core.

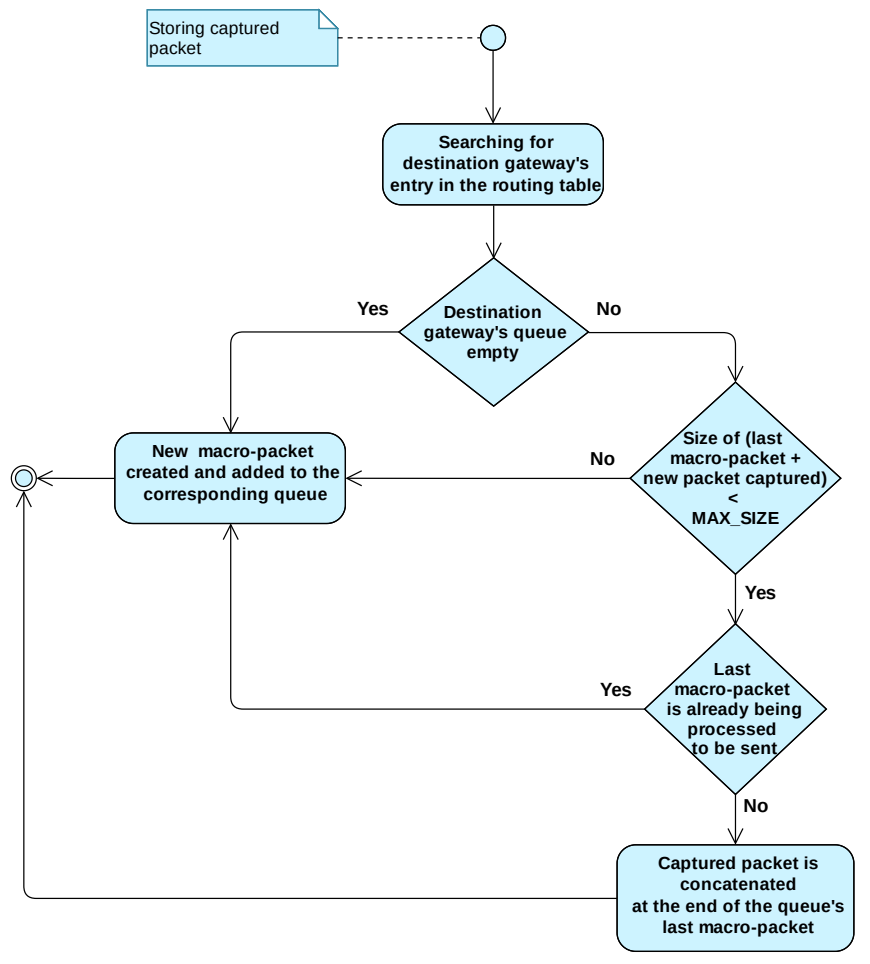

Figure 4. Procedure for storing a newly arrived IP packet at the gateway.

All these adjustments in the storing and managing of pending IP packets do not involve significant changes in how they must be retrieved from the queue. The only change is that the gateway that receives the Interest Datagram needs not only the sequence number of the macro-packet but also the identifier of the queue where it was stored (information included in the name of the Interest Datagram). The retrieving process itself is analogous to that taking place in the basic version of the protocol: the macro-packet, now identified by its queue and its sequence number, is retrieved from the queue and becomes the content of the Data packet sent to satisfy the corresponding Interest Datagram.

Finally, note that this improved version also complicates the process executed by the gateways when they receive a Data packet, since its content is no longer an individual IP packet but rather a set of concatenated datagrams. Thus, once the macro-packet is extracted from the Data packet, the gateway must separate the chained IP packets before sending them individually to their corresponding IP subnets. This task is accomplished by using the total size of the content of the Data packet and the individual size of each IP packet (which are obtained from their corresponding headers). The particular operation done at this point is detailed in Fig. 5. Thanks to this process, the extracted IP packets are sent one by one to their IP destination addresses until the end of the macro-packet is reached. 


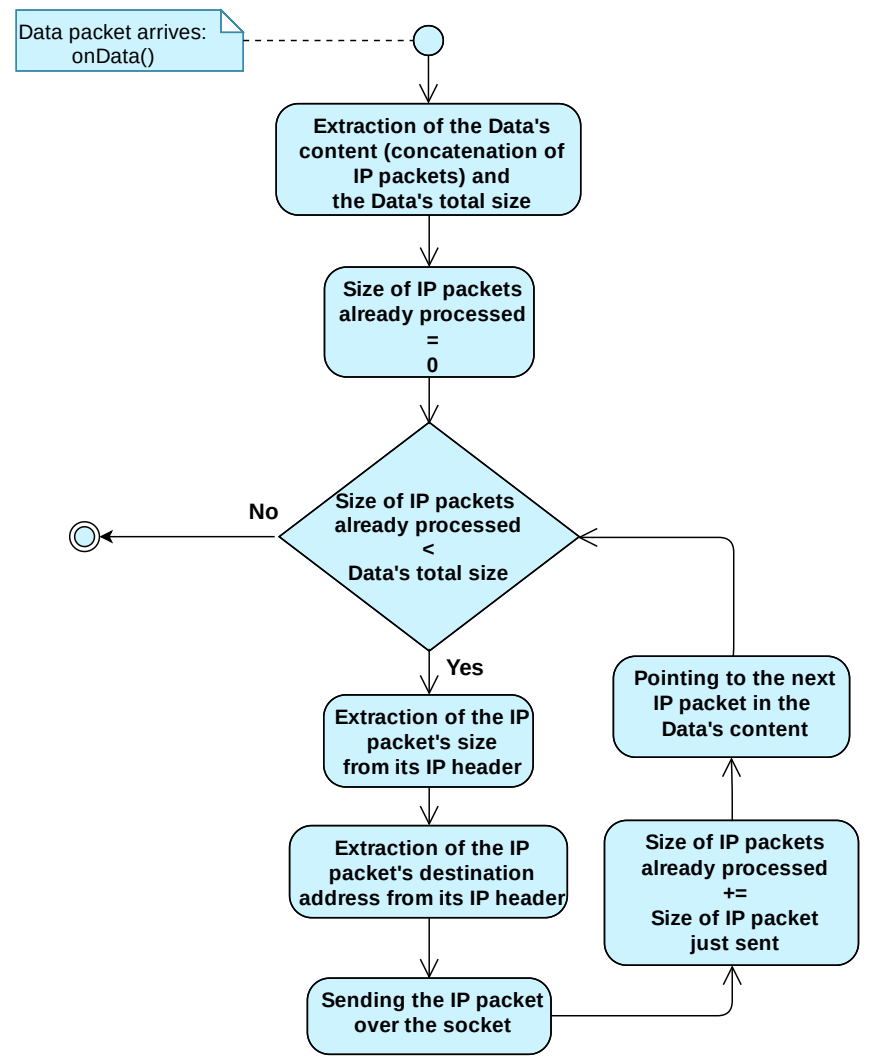

Figure 5. Procedure on arrival of a Data packet carrying IP traffic.

\section{Experimental Results}

Figure 2 shows the concrete scenario used to test our IP over NDN gateway with both the basic and the enhanced protocol versions. As the diagram illustrates, the system includes two independent IP subnets (subnet $A$ to the left and subnet $B$ to the right), both connected to a core composed of three routers implementing the NDN protocol. We have performed the same set of tests for both versions of the gateway protocol to compare their performance. We used the NFD daemon in the NDN routers. The official C++ NDN library (ndn-cxx [8]) was used to implement the main code of the gateway. For the link level connection between the three NDN routers we explored three different technologies: a) using Ethernet directly as the NDN face, ${ }^{3}$ operating both multicast and unicast faces with their corresponding MAC addresses; $b$ ) using the IPv6 support; and $c$ ) using an IPv4 configuration either with udp4 multicast interfaces or pointto-point interfaces. After configuring and implementing all these alternatives, we finally chose the IPv4 configuration with routes configured over UDP multicast faces due to its simplicity. In any case, note that, for our tests, the gateway

3. Generalization of the concept of network interface used in the NDN architecture. The term face refers to an abstraction of any communication channel that the NFD may use for forwarding NDN packets, since the exchange of packets is done not only over physical network interfaces but also directly between applications located in the same machine.

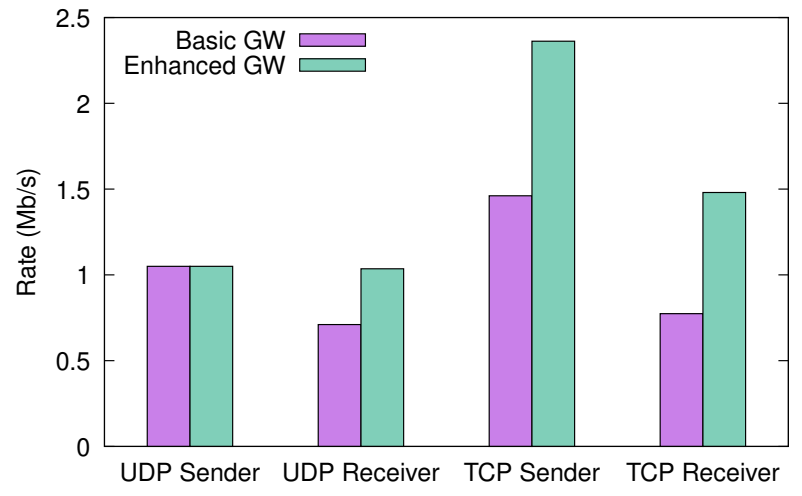

Figure 6. Transmission rates obtained in the iperf experiments.

performance does not depend on the link layer technology that much. The configuration of the routes over these faces was statically done using NFD configuration commands.

Initially, we performed some basic tests using the ping command, the Wireshark packet analyzer and the dissectwireshark tool (included in the $n d n$-tools) to verify that the capture, the transport and the delivery of IP packets between host $A$ and hostB were done properly.

Then, to gain confidence on the gateway implementation and to confirm that it was also capable of dealing with bursts and the transport of other protocols besides ICMP (the only one used by the ping command), we performed some additional experiments with iperf, a tool that can create data streams between two endpoints in one or both directions. ${ }^{4}$ With these experiments, we demonstrated that our gateway operates successfully even when the hosts transmit large amounts of data using different transport protocols such as TCP or UDP.

Additionally, we also used the iperf tests to analyze the performance provided by both the proposed forwarding protocols (the basic protocol and the enhanced one). Figure 6 shows the transmission rates obtained with UDP and TCP when using the basic gateway protocol or the improved one. ${ }^{5}$ As expected, the rate achieved with the improved version of the gateway is significantly higher. Note that the iperf UDP sender is unaware of packet losses and thus obtains the same throughput regardless of what happens to the transmitted traffic.

We also measured other important performance parameters, such as the jitter and the packet loss rate, when sending UDP traffic. Thus, we observed that the average jitter when using the improved version of the gateway is reduced from $13.8 \mathrm{~ms}$ to $8.6 \mathrm{~ms}$. Moreover, with the improved protocol, no packet losses were experienced, compared to the $7.5 \%$ packet loss rate measured with the basic one.

4. The tests with iperf were performed executing the server in hostA and the client in host $B$ and vice versa.

5. Each iperf experiment was repeated 10 times to obtain reliable measurements. The mean and the $95 \%$ confidence interval (CI) of the transmission rate at both the sender and receiver sides were calculated, but CIs are not shown in the graph since all of them are small and just clutter the figure. 
Finally, we performed a visual demonstration with video streaming. This is a good test to check the proper operation of our gateway, as both jitter and packet losses manifest clearly in the resulting reproduction. In particular, we ran a streaming application between hostA and hostB. In order to perform this test, ffmpeg was used to serve the simultaneous streaming videos while VLC clients were used to display them. ${ }^{6}$ Two specific tests were performed for each version of the gateway protocol: a) hostA serves a video while host $B$ displays it through a VLC client. Simultaneously, hostB serves a different video, which is displayed by hostA through a VLC client as well; $b$ ) host $B$ simultaneously serves two different videos and hostA displays both of them using two VLC clients. Both tests were recorded and, as expected, the results were consistent with those obtained using iperf. It can be clearly seen that, when the basic version of the gateway protocol is used, the video playback is choppy, stuttering continuously. In contrast, with the improved version, the video playback is smooth and experiences no pauses nor image corruption artifacts. Therefore, these tests show that multimedia data can be successfully transmitted through the gateways, with a good user experience when the improved protocol is used.

\section{Conclusions}

The main intention of this work was to contribute to adjust the current network layer to today's dominant use of the Internet as a content distribution network rather than as a communication one. For this, we have presented in this paper a novel design for IP over NDN gateways that includes a new forwarding protocol that enables the efficient and transparent transport of IP packets through an NDN network. The proposed gateway design has been implemented and evaluated in a real-world scenario using the latest available version of the NFD software and standard tools like iperf as well as multimedia traffic with the help of $V L C$. Performance results revealed that the developed tool is completely functional and that it can be successfully used to migrate from IP networks to NDN ones. Moreover, the analysis of these results, both quantitative (iperf results) and qualitative (streaming application results), shows that our IP over NDN gateway offers a good user experience thanks to the new efficient forwarding protocol proposed.

As future work, we will explore the following research areas:

1) We plan to use ChronoSync [18] when configuring the routing tables at the gateways. ChronoSync is a protocol employed to synchronize the state of a dataset among a distributed group of NDN users. Therefore, it would allow all the gateways in the network to automatically synchronize their routing tables instead of loading it individually from a local configuration file during their initialization.

6. Screen captures from the test can be visualized at the project web site at https://icarus.det.uvigo.es/ndnOverIP/.
2) We will also explore the possibility that our gateways use multicast traffic support, taking advantage of the innetwork storage that the NDN architecture inherently offers. Note that NDN routers can store Data packets in their Content Store temporarily, using it as a cache memory.

\section{Acknowledgments}

This publication is part of the research project PID2020-113240RB-I00, financed by MCIN/ AEI/10.13039/501100011033.

\section{References}

[1] D. Kutscher, B. Ohlman, and D. Oran, "Information-Centric Networking Research Group," Apr. 2012, publisher: Internet Research Task Force. [Online]. Available: https://irtf.org/icnrg

[2] L. Zhang, A. Afanasyev, J. Burke, V. Jacobson, K. Claffy, P. Crowley, C. Papadopoulos, L. Wang, and B. Zhang, "Named data networking," Computer Communication Review, vol. 44, no. 3, pp. 66-73, 2014.

[3] F. Fahrianto and N. Kamiyama, "The Dual-Channel IP-to-NDN Translation Gateway," in 2021 IEEE International Symposium on Local and Metropolitan Area Networks (LANMAN), Jul. 2021, pp. 1-2, iSSN: 1944-0375.

[4] _ "A Low-Cost IP-to-NDN Translation Gateway," in 2021 IEEE 22nd International Conference on High Performance Switching and Routing (HPSR), Jun. 2021, pp. 1-5, iSSN: 2325-5609.

[5] Named Data Networking Project, "NDN packet format specification," version 0.3. [Online]. Available: https://named-data.net/doc/ NDN-packet-spec/current/intro.html

[6] S. Junxiao, A. Afanasyev, D. Pesavento, D. Newberry, K. Schneider, and T. Liang, "NDNLPv2 - NFD." [Online]. Available: https: //redmine.named-data.net/projects/nfd/wiki/NDNLPv2

[7] "Named Data Networking Forwarding Daemon," Apr. 2021, original-date: 2014-01-27T07:43:57Z. [Online]. Available: https: //github.com/named-data/NFD

[8] A. Afanasyev, Y. Yu, J. Shi, J. Thompson, and Z. Zhu, "ndncxx: NDN C++ library with eXperimental eXtensions." [Online]. Available: https://named-data.net/doc/ndn-cxx/current/

[9] A. Afanasyev and P. Davide, "ndn-tools: NDN Essential Tools." [Online]. Available: https://github.com/named-data/ndn-tools

[10] D. Kulinski and J. Burke, "NDNVideo: Random-access Live and Prerecorded Streaming using NDN," UCLA, Technical Report NDN0007, Sep. 2012.

[11] C. Ghasemi, H. Yousefi, and B. Zhang, "Far Cry: Will CDNs Hear NDN's Call?" in Proceedings of the 7th ACM Conference on Information-Centric Networking, ser. ICN '20. New York, NY, USA: Association for Computing Machinery, Sep. 2020, pp. 89-98. [Online]. Available: https://doi.org/10.1145/3405656.3418708

[12] P. Gusev and J. Burke, "NDN-RTC: Real-Time Videoconferencing over Named Data Networking," in Proceedings of the 2nd ACM Conference on Information-Centric Networking, ser. ACMICN '15. New York, NY, USA: Association for Computing Machinery, Sep. 2015, pp. 117-126. [Online]. Available: https: //doi.org/10.1145/2810156.2810176

[13] D. Saxena, V. Raychoudhury, and C. Becker, "Implementation and Performance Evaluation of Name-based Forwarding Schemes in V-NDN," in Proceedings of the 18th International Conference on Distributed Computing and Networking, ser. ICDCN'17. New York, NY, USA: Association for Computing Machinery, Jan. 2017, pp. 1-4. [Online]. Available: https://doi.org/10.1145/3007748.3007766 
[14] F. Fahrianto and N. Kamiyama, "Comparison of Migration Approaches of ICN/NDN on IP Networks," in 2020 Fifth International Conference on Informatics and Computing (ICIC), Nov. 2020, pp. $1-7$.

[15] S. Luo, S. Zhong, and K. Lei, "IP/NDN: A multi-level translation and migration mechanism," in NOMS 2018 - 2018 IEEE/IFIP Network Operations and Management Symposium, Apr. 2018, pp. 1-5, iSSN: 2374-9709.

[16] I. Moiseenko and D. Oran, "TCP/ICN: Carrying TCP over Content Centric and Named Data Networks," in Proceedings of the 3rd ACM Conference on Information-Centric Networking, ser. ACM-ICN '16. New York, NY, USA: Association for Computing Machinery, Sep. 2016, pp. 112-121. [Online]. Available: https://doi.org/10.1145/2984356.2984357

[17] Tcpdump Group, "Libpcap." [Online]. Available: https://www. tcpdump.org/index.html\#documentation

[18] Z. Zhu and A. Afanasyev, "Let's ChronoSync: Decentralized dataset state synchronization in Named Data Networking," in 201321 st IEEE International Conference on Network Protocols (ICNP), Oct. 2013, pp. 1-10, iSSN: 1092-1648. 